

\title{
Nonlinear elliptic problems in weighted variable exponent Sobolev spaces by topological degree
}

\author{
Mustapha Ait Hammou* (iD) orcid.org/0000-0002-3930-3469 \\ El Houssine Azroul ${ }^{* *}$ \\ *University Sidi Mohamed Ben Abdellah, FSDM, Fez, Morocco. \\ $\checkmark$ mus.aithammou@gmail.com \\ **University Sidi Mohamed Ben Abdellah, FSDM, Fez, Morocco. \\ azroulelhoussine@gmail.com
}

Received: July 2018 | Accepted: March 2019

\section{Abstract:}

In this paper, we prove the existence of solutions for the nonlinear $p(\cdot)$-degenerate problems involving nonlinear operators of the form

- $\operatorname{div} a(x, \nabla u)=f(x, u, \nabla u)$ where $a$ and $f$ are Carathéodory functions satisfying some nonstandard growth conditions.

Keywords: Nonlinear elliptic equation; Weighted Sobolev spaces with variable exponent; Dirichlet problem.

MSC (2010): 35J20, 35J25.

\section{Cite this article as (IEEE citation style):}

M. Hammou and E. Azroul, "Nonlinear elliptic problems in weighted variable exponent Sobolev spaces by topological degree", Proyecciones (Antofagasta, On line), vol. 38, no. 4, pp. 733-751, Oct. 2019, doi: 10.22199/issn.0717-6279-2019. 04-0048. [Accessed dd-mm-yyyy].

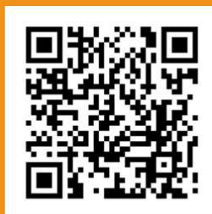

Article copyright: (c) 2019 Mustapha Ait Hammou, El Houssine Azroul. This is an open access article distributed under the terms of the Creative Commons Licence, which permits unrestricted use and distribution provided the original author and source are credited. 


\section{Introduction}

A class of nonlinear problems with variable exponents is a new search field which reflects a new type of physical phenomena. For a number of materials with inhomogenies, modeling using classical Lebesgue and Sobolev spaces has shown its limitation. For example, in the context of fluid mechanics, much attention has been given to the study of electrorological fluids that have the ability to change their mechanical properties when an electric field is applied to them. Recently, Rajagopal and M. Rúžička developed in [15] (see also [16]) a very interesting model for these fluids taking into account the delicate interaction between the electric field $E(x)$ and the moving liquid. The energy associated with this kind of problems is given by $\int_{\Omega}|\nabla u|^{p(x)} d x$ or rather by $\int_{\Omega} w(x)|\nabla u|^{p(x)} d x$. This type of energy also appears in the problems of elasticity [22].

The natural energy space in which such problems can be studied is the weighted variable exponent Sobolev space $W_{0}^{1, p(x)}(\Omega, w)$. For more information about these spaces and their properties, we refer to articles $[1,19,20]$ and references therein.

Let $\Omega \subset \mathbf{R}^{N}$ be an open bounded set with boundary $\partial \Omega$ of class $C^{1}$. In this paper we study the existence of solutions of the following nonlinear degenerated $p(x)$ elliptic problem

$$
\left\{\begin{array}{l}
-\operatorname{div} a(x, \nabla u)=f(x, u, \nabla u) \text { in } \Omega \\
u=0 \text { on } \partial \Omega .
\end{array}\right.
$$

where

$p \in \mathcal{C}_{+}(\Omega):=\left\{\right.$ measurable function $\left.h(\cdot): \bar{\Omega} \rightarrow \mathbf{R}^{+} / 1<h^{-} \leq h(x) \leq h^{+}<+\infty\right\}$,

whith

$$
h^{-}=\operatorname{essinf}\{h(x) / x \in \Omega\} \text { and } h^{+}=\operatorname{essup}\{h(x) / x \in \Omega\} .
$$

We also assume that $p$ satisfies the log-Hölder continuity condition, i.e. there is a constant $C>0$ such that for every $x, y \in \bar{\Omega}, x \neq y$ with $|x-y| \leq \frac{1}{2}$ one has

$$
|p(x)-p(y)| \leq \frac{C}{-\log |x-y|}
$$


The operator $A u \equiv-\operatorname{div} a(x, \nabla u)$ is a Leray-Lions operator defined on

$W_{0}^{1, p(x)}(\Omega, w)$, where $w$ is a weight function, satisfying some integrability conditions and $f$ is Carathéodory functions satisfying some nonstandard growth condition.

By studying the problem (1.1), we then extend both a class problems that involve Leray-Lions operators with variable exponents (see [2]) and another of degenerate problems that involve special weights $[2,7,10]$.

In this paper, the method used to treat the problem (1.1) is the topological degree which is widely used in the study of nonlinear equations, especially elliptic. Since the construction of the first topological degree by Brouwer in 1912 for continuous maps in finite dimensional Euclidean spaces [8], this theory has undergone several extensions and generalizations. Leray and Schauder generalized it in 1934 for compact operators in Banach spaces of infinite dimension [14]. Later, the theory was built by Berkovits and Mustonen [3, 4, 5, 6].

This paper is divided into three sections, organized as follows: in section 2 , we introduce some basic properties of the space $W^{1, p(x)}(\Omega, w)$ and an outline of Berkovits degree theory. In section 3, we prove the existence of solutions of (1.1).

\section{Mathematical Preliminaries}

\subsection{Weighted variable Lebesgue and Sobolev spaces}

In this subsection, we recall some definitions and fundamental properties of weighted variable Lebesgue and Sobolev spaces (see $[1,19,20]$ for more details).

Let $\Omega$ an open bounded subset of $\mathbf{R}^{N}(N \geq 1)$ and $p(\cdot) \in \mathcal{C}_{+}(\Omega)$ satisfying the log-Hölder continuity condition (1.2).

Definition 2.1. A function $w$ defined on $\Omega$ is called a weigh function if it is measurable and strictly positive a.e. in $\Omega$.

Let introduce the integrability conditions used on the framework of these spaces

$$
\begin{gathered}
w \in L_{l o c}^{1}(\Omega), \\
w^{*} \in L_{l o c}^{1}(\Omega),
\end{gathered}
$$


where $w^{*}(x)=w(x)^{1-p^{\prime}(x)}$ with $\frac{1}{p(x)}+\frac{1}{p^{\prime}(x)}=1$. We define the weighted variable exponent Lebesgue space as

$$
L^{p(x)}(\Omega, w)=\left\{u: \Omega \rightarrow \mathbf{R} \text { measurable, } \int_{\Omega}|u(x)|^{p(x)} w(x) d x<\infty\right\} .
$$

The space $L^{p(x)}(\Omega, w)$ endowed withe the norm :

$$
\|u\|_{p(x), w}=\inf \left\{\lambda>0, \int_{\Omega}\left|\frac{u(x)}{\lambda}\right|^{p(x)} w(x) d x \leq 1\right\}
$$

is a uniformly convex Banach space, and therefore reflexive. Let us denote

$$
I_{p, w}(u)=\int_{\Omega}|u|^{p(x)} w(x) d x, \quad \forall u \in L^{p(x)}(\Omega, w) .
$$

Note that if $w \equiv 1$, then $L^{p(x)}(\Omega, w)=L^{p(x)}(\Omega)$ and $I_{p, w}(u)=I_{p, 1}(u)=$ $\rho_{p(x)}$ (the convex modular, see $[11]$ ).

$L^{p^{\prime}(x)}\left(\Omega, w^{*}\right)$ is the conjugate space of $L^{p(x)}(\Omega, w)$.

By taking

$$
I_{p, w}(u)=\rho_{p(x)}\left(w^{\frac{1}{p(\cdot)}} u\right) \text { and }\left\|w^{\frac{1}{p(\cdot)}} u\right\|_{p(x)}=\|u\|_{p(x), w},
$$

we have, as in [11], the Hölder inequality

$\forall u \in L^{p(x)}(\Omega, w), \forall v \in L^{p^{\prime}(x)}\left(\Omega, w^{*}\right),\left|\int_{\Omega} u v d x\right| \leq\left(\frac{1}{p_{-}}+\frac{1}{p_{-}^{\prime}}\right)\|u\|_{p(x), w}\|v\|_{p^{\prime}(x), w^{*}}$. for all

We have also the continuous embedding $L^{p_{2}(x)}(\Omega, w) \hookrightarrow L^{p_{1}(x)}(\Omega, w)$

$p_{1}, p_{2} \in \mathcal{C}_{+}(\Omega)$ where $p_{1}(x) \leq p_{2}(x)$ a.e. $x \in \bar{\Omega}$.

We can prove also, by the identifications id, as in [11], the following important result

Proposition 2.2. for each $u \in L^{p(x)}(\Omega, w)$,

1. $\|u\|_{p(x), w}<1(\operatorname{resp} .=1$ or $>1) \Leftrightarrow I_{p, w}(u)<1($ resp. $=1$ or $>1)$,

2. $\|u\|_{p(x), w}>1 \Rightarrow\|u\|_{p(x), w}^{p_{-}} \leq I_{p, w}(u) \leq\|u\|_{p(x), w}^{p_{+}}$,

$$
\|u\|_{p(x), w}<1 \Rightarrow\|u\|_{p(x), w}^{p_{+}} \leq I_{p, w}(u) \leq\|u\|_{p(x), w}^{p_{-}},
$$


3. $I_{p, w}(u) \leq\|u\|_{p(x), w}^{p_{+}}+\|u\|_{p(x), w}^{p_{-}}$,

4. $\|u\|_{p(x), w} \leq I_{p, w}(u)+1$,

5. $\|u\|_{p(x), w} \rightarrow 0 \Leftrightarrow I_{p, w}(u) \rightarrow 0$ and $\|u\|_{p(x), w} \rightarrow \infty \Leftrightarrow I_{p, w}(u) \rightarrow \infty$.

The weighted variable exponent Sobolev space is defined by

$$
W^{1, p(x)}(\Omega, w)=\left\{u \in L^{p(x)}(\Omega) \text { and }|\nabla u| \in L^{p(x)}(\Omega, w)\right\} \text {. }
$$

equipped with the following norm

$$
\|u\|_{1, p(x), w}=\|u\|_{p(x)}+\|\nabla u\|_{p(x), w} \quad \text { for all } u \in W^{1, p(x)}(\Omega, w) .
$$

We denote by $W_{0}^{1, p(x)}(\Omega, w)$ the closure of $C_{0}^{\infty}(\Omega)$ in $W^{1, p(x)}(\Omega, w)$ with respect to the norm $\|u\|_{1, p(x), w}$, we have the following compact embedding

Proposition 2.3. [13, Proposition 2.3.] $W_{0}^{1, p(x)}(\Omega, w) \hookrightarrow \hookrightarrow L^{p(x)}(\Omega)$.

The dual space of $W_{0}^{1, p(\cdot)}(\Omega, w)$, denoted $W^{-1, p^{\prime}(\cdot)}\left(\Omega, w^{*}\right)$, is equipped with the norm

$$
\|v\|_{-1, p^{\prime}(x), w^{*}}=\inf \left\{\left\|v_{0}\right\|_{p^{\prime}(x), w^{*}}+\sum_{i=1}^{N}\left\|v_{i}\right\|_{p^{\prime}(x), w^{*}}\right\}
$$

where the infinimum is taken on all possible decompositions $v=v_{0}-\operatorname{div} F$.

Remark 2.4. [19, Theorem 7 and Corollary 2]The Poincaré inequality holds for the weighted Sobolev spaces $W_{0}^{1, p(\cdot)}(\Omega, w)$. In particular, this space has a norm $\|\cdot\|_{1, p(x)}$ given by

$$
\|u\|_{1, p(x)}=\|\nabla u\|_{p(x), w} \text { for all } u \in W_{0}^{1, p(\cdot)}(\Omega, w),
$$

which equivalent to $\|\cdot\|_{1, p(x), w}$. 


\subsection{An outline of Berkovits degree theory}

Let $X$ be a real separable reflexive Banach space with dual $X^{*}$ and with continuous pairing $\langle.,\rangle,. \Omega$ be a nonempty subset of $X$ and $Y$ be a real Banach space. The symbol $\rightarrow(\rightarrow)$ stands for strong (weak) convergence.

In this subsection, we give an outline of the Berkovits degree we will use to study the problem (1.1). We begin by defining the appropriate operator classes that Berkovits used to build this degree. The reader can find the definition of these classes in $[3,4,5,6]$.

We recall that a mapping $F: \Omega \subset X \rightarrow Y$ is

- bounded, if it takes any bounded set into a bounded set.

- demicontinuous, if for any $\left(u_{n}\right) \subset \Omega, u_{n} \rightarrow u$ implies $F\left(u_{n}\right) \rightarrow F(u)$.

- compact if it is continuous and the image of any bounded set is relatively compact.

A mapping $F: \Omega \subset X \rightarrow X^{*}$ is said to be

- of class $\left(S_{+}\right)$, if for any $\left(u_{n}\right) \subset \Omega$ with $u_{n} \rightarrow u$ and limsup $\left\langle F u_{n}, u_{n}-\right.$ $u\rangle \leq 0$, we have $u_{n} \rightarrow u$.

- quasimonotone, if for any $\left(u_{n}\right) \subset \Omega$ with $u_{n} \rightarrow u$, we have $\limsup \left\langle F u_{n}, u_{n}-u\right\rangle \geq 0$.

For any operator $F: \Omega \subset X \rightarrow X$ and any bounded operator $T: \Omega_{1} \subset$ $X \rightarrow X^{*}$ such that $\Omega \subset \Omega_{1}$, we say that $F$ is of class $\left(S_{+}\right)_{T}$, if for any $\left(u_{n}\right) \subset \Omega$ with $u_{n} \rightarrow u, y_{n}:=T u_{n} \rightarrow y$ and $\limsup \left\langle F u_{n}, y_{n}-y\right\rangle \leq 0$, we have $u_{n} \rightarrow u$.

Let $\mathcal{O}$ be the collection of all bounded open set in $X$. For any $\Omega \subset X$, we consider the following classes of operators:

$$
\begin{aligned}
\mathcal{F}_{1}(\Omega) & :=\left\{F: \Omega \rightarrow X^{*} \mid F \text { is bounded, demicontinuous and of class }\left(S_{+}\right)\right\}, \\
\mathcal{F}_{T, B}(\Omega) & :=\left\{F: \Omega \rightarrow X \mid F \text { is bounded, demicontinuous and of class }\left(S_{+}\right)_{T}\right\}, \\
\mathcal{F}_{T}(\Omega) & :=\left\{F: \Omega \rightarrow X \mid F \text { is demicontinuous and off class }\left(S_{+}\right)_{T}\right\}, \\
\mathcal{F}_{B}(X) & :=\left\{F \in \mathcal{F}_{T, B}(\bar{G}) \mid G \in \mathcal{O}, \mathrm{T} \in \mathcal{F}_{1}(\overline{\mathrm{G}})\right\} .
\end{aligned}
$$

Here, $T \in \mathcal{F}_{1}(\bar{G})$ is called an essential inner map to $F$. 
Definition 2.5. Let $G$ be a bounded open subset of a real reflexive Banach space $X, T \in \mathcal{F}_{1}(\bar{G})$ be continuous and let $F, S \in \mathcal{F}_{T}(\bar{G})$. We define a class $\mathcal{H}_{T}(\bar{G})$ (called a class of admissible affine homotopy with the common continuous essential inner map $T$ ) as the class of affine homotopy $H$ : $[0,1] \times \bar{G} \rightarrow X$ defined by

$$
H(t, u):=(1-t) F u+t S u \text { for }(t, u) \in[0,1] \times \bar{G}
$$

Remark 2.6. [4]

$$
\mathcal{H}_{T}(\bar{G}) \subset\left(S_{+}\right)_{T}
$$

We introduce the topological degree for the class $\mathcal{F}_{B}(X)$ due to Berkovits $[4]$.

Theorem 2.7. There exists a unique degree function

$$
d:\left\{(F, G, h) \mid G \in \mathcal{O}, T \in \mathcal{F}_{1}(\bar{G}), F \in \mathcal{F}_{T, B}(\bar{G}), h \notin F(\partial G)\right\} \rightarrow \mathbf{Z}
$$

that satisfies the following properties

1. (Existence) if $d(F, G, h) \neq 0$, then the equation

$$
F u=h
$$

has a solution in $G$.

2. (Additivity) Let $F \in \mathcal{F}_{T, B}(\bar{G})$. If $G_{1}$ and $G_{2}$ are two disjoint open subset of $G$ such that $h \notin F\left(\bar{G} \backslash\left(G_{1} \cup G_{2}\right)\right)$, then we have

$$
d(F, G, h)=d\left(F, G_{1}, h\right)+d\left(F, G_{2}, h\right)
$$

3. (Homotopy invariance) If $H \in \mathcal{H}_{T}(\bar{G})$ is bounded and $h:[0,1] \rightarrow X$ is a continuous path in $X$ such that $h(t) \notin H(t, \partial G)$ for all $t \in[0,1]$, then the value of $d(H(t,), G,. h(t))$ is constant for all $t \in[0,1]$.

4. (Normalization) For any $h \in G$, we have

$$
d(I, G, h)=1
$$

where I denotes the identity operator. 


\section{Main result}

\subsection{Basic assumptions and technical lemmas}

Throughout the paper, we assume that the following assumptions hold. Let $\Omega$ be a bounded open set of $\mathbf{R}^{N}(N \geq 1)$ and $p(\cdot) \in \mathcal{C}_{+}(\bar{\Omega})$. And let $w$ a weight function in $\Omega$ which satisfies the integrability conditions (2.1) and (2.2).

The function $a: \Omega \times \mathbf{R}^{N} \rightarrow \mathbf{R}^{N}$ is a Carathéodory function satisfying the following conditions: For all $\xi \in \mathbf{R}^{N}$ and for almost every $x \in \Omega$,

$$
\begin{gathered}
|a(x, \xi)| \leq \beta w^{\frac{1}{p(x)}}\left(k(x)+w^{\frac{1}{p^{\prime}(x)}}|\xi|^{p(x)-1}\right), \\
{[a(x, \xi)-a(x, \eta)](\xi-\eta)>0 \quad \forall \xi \neq \eta,} \\
a(x, \xi) \xi \geq \alpha w(x)|\xi|^{p(x)},
\end{gathered}
$$

where $k(x)$ is a positive function in $L^{p^{\prime}(x)}(\Omega)$ and $\alpha$ and $\beta$ are a positive constants.

Let $f(x, s, \xi): \Omega \times \mathbf{R} \times \mathbf{R}^{N} \rightarrow \mathbf{R}$ be a Carathéodory function such that for a.e. $x \in \Omega$ and for all $s \in \mathbf{R}, \xi \in \mathbf{R}^{N}$, the growth condition

$$
|f(x, s, \xi)| \leq \gamma w^{\frac{1}{p(x)}}\left(h(x)+w^{\frac{1}{p^{\prime}(x)}}|s|^{q(x)-1}+w^{\frac{1}{p^{\prime}(x)}}|\xi|^{q(x)-1}\right)
$$

for a.e. $x \in \Omega$ and all $(s, \xi) \in \mathbf{R} \times \mathbf{R}^{N}$, where $\gamma$ is a positive constant, $h$ is a positive function in $L^{p^{\prime}(x)}(\Omega)$ and $1<q^{-} \leq q(x) \leq q^{+}<p^{-}$.

Lemma 3.1. [13] Let $g \in L^{r(x)}(\Omega, w)$ and $g_{n} \in L^{r(x)}(\Omega, w)$ with $\left\|g_{n}\right\|_{r(x), w} \leq$ $C$ for $1<r(x)<\infty$. If $g_{n}(x) \rightarrow g(x)$ a.e on $\Omega$, then $g_{n} \rightarrow g$ in $L^{r(x)}(\Omega, w)$.

Let define the operator $A: W_{0}^{1, p(x)}(\Omega, w) \rightarrow W^{-1, p^{\prime}(x)}\left(\Omega, w^{*}\right)$ by

$$
A u=-\operatorname{diva}(x, \nabla u)
$$

then,

$$
\langle A u, v\rangle=\int_{\Omega} a(x, \nabla u) \nabla v d x,
$$

for all $v$ in $W_{0}^{1, p(x)}(\Omega, w)$.

Lemma 3.2. If the assumptions (3.1)-(3.3) holds, then $A$ is bounded, continuous, coercive and of class $\left(S_{+}\right)$. 


\section{Proof. Step 1:}

Let's show that the operator $A$ is bounded.

By using the Hölder's inequality (2.4), we have for all $u, v \in W_{0}^{1, p(x)}(\Omega, w)$

$$
\begin{aligned}
|\langle A u, v\rangle| & =\left|\int_{\Omega} a(x, \nabla u) \nabla v d x\right| \\
& =\left|\int_{\Omega} a(x, \nabla u) w(x)^{\frac{-1}{p(x)}} \cdot \nabla v w(x)^{\frac{1}{p(x)}} d x\right| \\
& \leq C\left\|a(x, \nabla u) w(x)^{\frac{-1}{p(x)}}\right\|_{p^{\prime}(x)} \cdot\left\|\nabla v w(x)^{\frac{1}{p(x)}}\right\|_{p(x)} .
\end{aligned}
$$

Thanks to (2.1), (3.1) and Proposition 2.2 we can easily prove that

$$
\left\|a(x, \nabla u) w(x)^{\frac{-1}{p(x)}}\right\|_{p^{\prime}(x)} \text { is bounded for all } u \in W_{0}^{1, p(x)}(\Omega, w) \text {. Therefore }
$$

$$
\begin{aligned}
|\langle A u, v\rangle| & \leq \text { const }\left\|\nabla v w(x)^{\frac{1}{p(x)}}\right\|_{p(x)} \\
& =\operatorname{const}\|\nabla v\|_{p(x), w} \\
& =\text { const }\|v\|_{1, p(x), w},
\end{aligned}
$$

which means that the operator $A$ is bounded.

\section{Step 2:}

To show that $A$ is continuous, let $u_{n} \rightarrow u$ in $W_{0}^{1, p(x)}(\Omega, w)$. Then $\nabla u_{n} \rightarrow \nabla u$ in $L^{p(x)}(\Omega, w)$. Hence there exist a subsequence $\left(u_{k}\right)$ of $\left(u_{n}\right)$ and measurable function $g$ in $\left(L^{p(x)}(\Omega, w)\right)^{N}$ such that

$$
\nabla u_{k}(x) \rightarrow \nabla u(x) \text { and }\left|\nabla u_{k}(x)\right| \leq g(x)
$$

for a.e. $x \in \Omega$ and all $k \in \mathbf{N}$. Since $a$ satisfies the Carathodory condition, we have

$$
a\left(x, \nabla u_{k}(x)\right) \rightarrow a(x, \nabla u(x)) \text { a.e. } x \in \Omega .
$$

and from (3.1), we get

$$
\left|a\left(x, \nabla u_{k}(x)\right)\right| \leq \beta w^{\frac{1}{p(x)}}\left(k(x)+w^{\frac{1}{p^{\prime}(x)}}|g(x)|^{p(x)-1}\right)
$$

for a.e. $x \in \Omega$ and for all $k \in \mathbf{N}$. Since

$\int_{\Omega}\left[w^{\frac{1}{p(x)}}\left(k(x)+w^{\frac{1}{p^{\prime}(x)}}|g(x)|^{p(x)-1}\right)\right]^{p^{\prime}(x)} w^{*}(x) d x \leq C \int_{\Omega}\left(|k(x)|^{p^{\prime}(x)}+w|g(x)|^{p(x)}\right)<\infty$, 
(because $k \in L^{p^{\prime}(x)}(\Omega)$ and $\left.g \in\left(L^{p(x)}(\Omega, w)\right)^{N}\right)$, then

$$
x \longmapsto \beta w^{\frac{1}{p(x)}}\left(k(x)+w^{\frac{1}{p^{\prime}(x)}}|g(x)|^{p(x)-1}\right) \in L^{p^{\prime}(x)}\left(\Omega, w^{*}\right),
$$

and thanks to the equality

$$
I_{p^{\prime}, w^{*}}\left(a\left(x, \nabla u_{k}\right)-a(x, \nabla u)\right)=\int_{\Omega}\left|a\left(x, \nabla u_{k}(x)\right)-a(x, \nabla u(x))\right|^{p^{\prime}(x)} w^{*}(x) d x,
$$

the dominated convergence theorem and the equivalence (5) of Proposition 2.2 allow us to assert that

$$
a\left(x, \nabla u_{k}\right) \rightarrow a(x, \nabla u) \text { in } L^{p^{\prime}(x)}\left(\Omega, w^{*}\right) .
$$

Thus the entire sequence $a\left(x, \nabla u_{n}\right)$ converges to $a(x, \nabla u)$ in $L^{p^{\prime}(x)}\left(\Omega, w^{*}\right)$.

Then, $\forall v \in W_{0}^{1, p(x)}(\Omega, w) ;\left\langle A u_{n}, v\right\rangle \rightarrow\langle A u, v\rangle$, which implies that the operator $A$ is continuous.

\section{Step 3}

In this step, we show that $A$ is coercive. For that Let $v \in W_{0}^{1, p(x)}(\Omega, w)$, We have from (3.3), the Proposition 2.2 and Remark 2.4

$$
\frac{<A v, v>}{\|v\|_{1, p(x), w}} \geq \frac{C}{\|v\|_{1, p(x)}} \alpha I_{p, w}(\nabla v) \geq C^{\prime}\|v\|_{1, p(x)}^{r}
$$

for some $r>1$. Therefore

$$
\frac{<A v, v>}{\|v\|_{1, p(x), w}} \longrightarrow \infty \quad \text { as }\|v\|_{1, p(x), w} \rightarrow \infty
$$

So, $A$ is coercive.

\section{Step 4}

It remains to show that the operator $A$ is of class $\left(S_{+}\right)$. Let $\left(u_{n}\right)_{n}$ be a sequence in $W_{0}^{1, p(x)}(\Omega, w)$ such that

$$
\left\{\begin{array}{l}
u_{n} \rightarrow u \quad \text { in } W_{0}^{1, p(x)}(\Omega, w) \\
\limsup _{k \rightarrow \infty}\left\langle A u_{n}, u_{n}-u\right\rangle \leq 0
\end{array}\right.
$$

We will prove that

$$
u_{n} \longrightarrow u \text { in } W_{0}^{1, p(x)}(\Omega, w) .
$$


Since $u_{n} \rightarrow u$ in $W_{0}^{1, p(x)}(\Omega, w)$, then $\left(u_{n}\right)_{n}$ is a bounded sequence in $W_{0}^{1, p(x)}(\Omega, w)$. By using the Propositions 2.2 and 2.3 , there exist a subsequence still denoted by $\left(u_{n}\right)_{n}$ such that

$$
\begin{gathered}
u_{n} \rightarrow u \text { in } W_{0}^{1, p(x)}(\Omega, w), \\
u_{n} \rightarrow u \text { in } L^{p(x)}(\Omega) \text { and a.e in } \Omega .
\end{gathered}
$$

From (3.2) and (3.6), we have

$$
\lim _{n \rightarrow \infty}\left\langle A u_{n}, u_{n}-u\right\rangle=\lim _{n \rightarrow \infty}\left\langle A u_{n}-A u, u_{n}-u\right\rangle=0 .
$$

Let $D_{n}=a\left(x, \nabla u_{n}\right) \cdot \nabla\left(u_{n}-u\right)$. By (3.7) $D_{n} \rightarrow 0$ in $L^{1}(\Omega)$ and for a subsequence $D_{n} \rightarrow 0$ a.e. in $\Omega$.

Since $u_{n} \rightarrow u$ in $W_{0}^{1, p(x)}(\Omega, w)$ and a.e in $\Omega$, there exists a subset $B$ of $\Omega$, of zero measure, such that for $x \in \Omega \backslash B,|u(x)|<\infty,|\nabla u(x)|<\infty$, $k(x)<\infty, u_{n}(x) \rightarrow u(x), D_{n}(x) \rightarrow 0$.

Defining $\xi_{n}=\nabla u_{n}(x), \xi=\nabla u(x)$, we have

$\mathrm{D}_{n}(x)=a\left(x, \xi_{n}\right) \cdot\left(\xi_{n}-\xi\right)$

$=a\left(x, \xi_{n}\right) \xi_{n}-a\left(x, \xi_{n}\right) \xi$

$\geq \alpha w(x)\left|\xi_{n}\right|^{p(x)}-\beta w(x)^{\frac{1}{p(x)}}\left(k(x)+w(x)^{\frac{1}{p^{\prime}(x)}}\left|\xi_{n}\right|^{p(x)-1}\right)|\xi|$

$\geq \alpha\left|\xi_{n}\right|^{p(x)}-C_{x}\left[1+\left|\xi_{n}\right|^{p(x)-1}\right]$,

where $C_{x}$ is a constant which depends on $x$, but does not depend on $n$. Since

$u_{n}(x) \rightarrow u(x)$ we have $\left|u_{n}(x)\right| \leq M_{x}$, where $M_{x}$ is some positive constant. Then by a standard argument $\left|\xi_{n}\right|$ is bounded uniformly with respect to $n$, we deduce that

$$
D_{n}(x) \geq\left|\xi_{n}\right|^{p(x)}\left(\alpha-\frac{C_{x}}{\left|\xi_{n}\right|^{p(x)}}-\frac{C_{x}}{\left|\xi_{n}\right|}\right)
$$

If $\left|\xi_{n}\right| \rightarrow \infty$ (for a subsequence), then $D_{n}(x) \rightarrow \infty$ which gives a contradiction. Let now $\xi^{*}$ be a cluster point of $\xi_{n}$. We have $\left|\xi^{*}\right|<\infty$ and by the continuity of $a$ we obtain

$$
a\left(x, \xi^{*}\right) \cdot\left(\xi^{*}-\xi\right)=0 .
$$

In view of $(3.2)$, we have $\xi^{*}=\xi$, which implies that

$$
\nabla u_{n}(x) \rightarrow \nabla u(x) \quad \text { a.e.in } \Omega .
$$


Since the sequence $a\left(x, \nabla u_{n}\right)$ is bounded in $\left(L^{p^{\prime}(x)}\left(\Omega, w^{*}\right)\right)^{N}$, and

$$
\begin{array}{r}
a\left(x, \nabla u_{n}\right) \rightarrow a(x, \nabla u) \text { a.e. in } \Omega \text {, then by Lemma } 3.1 \text { we get } \\
a\left(x, \nabla u_{n}\right) \rightarrow a(x, \nabla u) \quad \text { in }\left(L^{p^{\prime}(x)}\left(\Omega, w^{*}\right)\right)^{N} \text { a.e. in } \Omega .
\end{array}
$$

We set $\bar{y}_{n}=a\left(x, \nabla u_{n}\right) \nabla u_{n}$ and $\bar{y}=a(x, \nabla u) \nabla u$. We can write

$$
\bar{y}_{n} \rightarrow \bar{y} \quad \text { in } L^{1}(\Omega) .
$$

We have

$$
a\left(x, \nabla u_{n}\right) \cdot \nabla u_{n} \geq \alpha w(x)\left|\nabla u_{n}\right|^{p(x)} .
$$

Let $z_{n}=w\left|\nabla u_{n}\right|^{p(.)}, z=w|\nabla u|^{p(.)}, y_{n}=\frac{\bar{y}_{n}}{\alpha}$, and $y=\frac{\bar{y}}{\alpha}$. By Fatou's lemma,

$$
\int_{\Omega} 2 y d x \leq \liminf _{n \rightarrow \infty} \int_{\Omega} y+y_{n}-\left|z_{n}-z\right| d x
$$

i.e., $0 \leq-\limsup _{n \rightarrow \infty} \int_{\Omega}\left|z_{n}-z\right| d x$. Then

$$
0 \leq \liminf _{n \rightarrow \infty} \int_{\Omega}\left|z_{n}-z\right| d x \leq \limsup _{n \rightarrow \infty} \int_{\Omega}\left|z_{n}-z\right| d x \leq 0,
$$

this implies

$$
\nabla u_{n} \rightarrow \nabla u \quad \text { in }\left(L^{p(x)}(\Omega, w)\right)^{N} .
$$

Hence $u_{n} \rightarrow u$ in $W_{0}^{1, p(x)}(\Omega, w)$, which completes the proof.

Lemma 3.3. Let $S: W_{0}^{1, p(x)}(\Omega, w) \rightarrow W^{-1, p^{\prime}(x)}\left(\Omega, w^{*}\right)$ be the operator setting by

$$
\langle S u, v\rangle=-\int_{\Omega} f(x, u, \nabla u) v d x, \quad \forall u, v \in W_{0}^{1, p(x)}(\Omega, w) .
$$

If the assumption (3.4) holds, then $S$ is compact.

Proof. Let us define an operator $\phi: W_{0}^{1, p(x)}(\Omega, w) \rightarrow L^{p^{\prime}(x)}\left(\Omega, w^{*}\right)$ by

$$
\phi u(x):=-f(x, u, \nabla u) \text { for } u \in W_{0}^{1, p(x)}(\Omega, w) \text { and } x \in \Omega .
$$

We begin by showing that $\phi$ is bounded and continuous.

For each $u \in W_{0}^{1, p(x)}(\Omega, w)$, we have by the growth condition (3.4), the inequalities (3) and (4) of Proposition 2.2 that 


$$
\begin{aligned}
\|\phi u\|_{p^{\prime}(x), w^{*}} & \leq I_{p^{\prime}, w}(\phi u)+1 \\
& =\int_{\Omega} \mid f\left(x, u(x),\left.\nabla u(x)\right|^{p^{\prime}(x)} w^{*}(x) d x+1\right. \\
& \leq \operatorname{const}\left(\rho_{p^{\prime}(x)}(h)+I_{r, w}(u)+I_{r, w}(\nabla u)\right)+1 \\
& \leq \operatorname{const}\left(\|h\|_{p^{\prime}(x)}^{p^{+}}+\|h\|_{p^{\prime}(x)}^{p^{\prime}}+\|u\|_{r(x), w}^{r^{+}}+\|u\|_{r(x), w}^{r^{-}}\right. \\
& \left.+\|\nabla u\|_{r(x), w}^{r^{+}}+\|\nabla u\|_{r(x), w}^{r^{-}}\right)+1
\end{aligned}
$$

where $r(x)=(q(x)-1) p^{\prime}(x)<p(x)$. By the continuous embedding $L^{p(x)} \hookrightarrow$ $L^{r(x)}$ and the Poincaré inequality, we have

$$
\|\phi u\|_{p^{\prime}(x), w^{*}} \leq \operatorname{const}\left(\|h\|_{p^{\prime}(x)}^{p^{+}}+\|h\|_{p^{\prime}(x)}^{p^{-}}+\|u\|_{1, p(x), w}^{r^{+}}+\|u\|_{1, p(x), w}^{r^{-}}\right)+1
$$

Which means that that $\phi$ is bounded on $W_{0}^{1, p(x)}(\Omega, w)$.

To show that $\phi$ is continuous, let $u_{n} \rightarrow u$ in $W_{0}^{1, p(x)}(\Omega, w)$. Then $u_{n} \rightarrow u$ in $L^{p(x)}(\Omega)$ and $\nabla u_{n} \rightarrow \nabla u$ in $\left(L^{p(x)}(\Omega, w)\right)^{N}$. We can thus extract from $\left(u_{n}\right)$ a subsequence $\left(u_{k}\right)$ and there exist measurable functions $H$ in $L^{p(x)}(\Omega)$ and $G$ in $\left(L^{p(x)}(\Omega, w)\right)^{N}$ such that

$$
\begin{gathered}
u_{k}(x) \rightarrow u(x) \text { and } \nabla u_{k}(x) \rightarrow \nabla u(x), \\
\left|u_{k}(x)\right| \leq H(x) \text { and }\left|\nabla u_{k}(x)\right| \leq|G(x)|
\end{gathered}
$$

for a.e. $x \in \Omega$ and all $k \in \mathbf{N}$. Since $f$ satisfies the Carathodory condition, we get

$$
f\left(x, u_{k}(x), \nabla u_{k}(x)\right) \rightarrow f(x, u(x), \nabla u(x)) \text { a.e. } x \in \Omega .
$$

From (3.4), it follows that

$$
\left|f\left(x, u_{k}(x), \nabla u_{k}(x)\right)\right| \leq \gamma w^{\frac{1}{p(x)}}\left(h(x)+w^{\frac{1}{p^{\prime}(x)}}|H(x)|^{q(x)-1}+w^{\frac{1}{p^{\prime}(x)}}|G(x)|^{q(x)-1}\right)
$$

for a.e. $x \in \Omega$ and for all $k \in \mathbf{N}$.

Since

$$
w^{\frac{1}{p(x)}}\left(h(x)+w^{\frac{1}{p^{\prime}(x)}}|H(x)|^{q(x)-1}+w^{\frac{1}{p^{\prime}(x)}}|G(x)|^{q(x)-1}\right) \in L^{p^{\prime}(x)}\left(\Omega, w^{*}\right),
$$

and by the equality

$$
I_{p^{\prime}, w^{*}}\left(\phi u_{k}-\phi u\right)=\int_{\Omega}\left|f\left(x, u_{k}(x), \nabla u_{k}(x)\right)-f(x, u(x), \nabla u(x))\right|^{p^{\prime}(x)} w^{*}(x) d x,
$$

we can conclude with the dominated convergence theorem and the equivalence (5) of the Proposition 2.2 that

$$
\phi u_{k} \rightarrow \phi u \text { in } L^{p^{\prime}(x)}\left(\Omega, w^{*}\right) .
$$


Thus, the entire sequence $\left(\phi u_{n}\right)$ converges $\phi u$ in $L^{p^{\prime}(x)}\left(\Omega, w^{*}\right)$. This means that $\phi$ is continuous.

Since the embedding $I: W_{0}^{1, p(x)}(\Omega, w) \hookrightarrow \hookrightarrow L^{p(x)}(\Omega, w)$ is compact, its adjoint operator $I^{*}: L^{p^{\prime}(x)}\left(\Omega, w^{*}\right) \rightarrow W^{-1, p^{\prime}(x)}\left(\Omega, w^{*}\right)$ is also compact. Finally, the composition

$$
S=I^{*} o \phi: W_{0}^{1, p(x)}(\Omega, w) \rightarrow W^{-1, p^{\prime}(x)}\left(\Omega, w^{*}\right)
$$

is compact.

Lemma 3.4. [4, Lemma 2.2 and 2.4] Let $G$ be a bounded open set in a real reflexive Banach space $X$. Suppose that $T \in \mathcal{F}_{1}(\bar{G})$ is continuous and $S: D_{S} \subset X^{*} \rightarrow X$ is demicontinuous such that $T(\bar{G}) \subset D_{S}$. Then the following statements hold:

- If $S$ is quasimonotone, then $I+S o T \in \mathcal{F}_{T}(\bar{G})$.

- If $S$ is of class $\left(S_{+}\right)$, then $S o T \in \mathcal{F}_{T}(\bar{G})$.

\subsection{Existence of weak solution}

Let us first define the weak solution of problem (1.1).

Definition 3.5. A weak solution of the problem (1.1) is a measurable function $u \in W_{0}^{1, p(x)}(\Omega, w)$ such that

$$
\int_{\Omega} a(x, \nabla u) \nabla v d x=\int_{\Omega} f(x, u, \nabla u) v d x
$$

for all $v \in W_{0}^{1, p(x)}(\Omega, w)$.

The main result of this paper is the following theorem.

Theorem 3.6. If the assumptions (3.1)-(3.4) holds, then the problem (1.1) admits at least one weak solution.

Proof. Let $A$ and $S: W_{0}^{1, p(x)}(\Omega, w) \rightarrow W^{-1, p^{\prime}(x)}\left(\Omega, w^{*}\right)$ be as in (3.5) and Lemma 3.3 respectively. Then $u \in W_{0}^{1, p(x)}(\Omega, w)$ is a weak solution of (1.1) if and only if

$$
A u=-S u
$$


Since the operator $A$ is strictly monotone (by assumbtion (3.2)), thanks to the properties seen in Lemma 3.2 and in view of Minty-Browder Theorem (see [21], Theorem 26A), the inverse operator $T:=A^{-1}: W^{-1, p^{\prime}(x)}\left(\Omega, w^{*}\right) \rightarrow$ $W_{0}^{1, p(x)}(\Omega, w)$ is bounded, continuous and of class $\left(S_{+}\right)$. Moreover, note from Lemma 3.3 that the operator $S$ is bounded, continuous and quasimonotone.

Therefore, equation (3.8) is equivalent to

$$
u=T v \text { and } v+S o T v=0 .
$$

To solve equation (3.9), we will apply the theory of Berkovits degree seen in Theorem 2.7. For this, we will first show that the set

$$
B:=\left\{v \in W^{-1, p^{\prime}(x)}\left(\Omega, w^{*}\right) \mid v+t S o T v=0 \text { for some } t \in[0,1]\right\}
$$

is bounded.

Indeed, let $v \in B$ and set $u:=T v$, then $\|T v\|_{1, p(x), w}=\|\nabla u\|_{p(x), w}$.

- If $\|\nabla u\|_{p(x), w} \leq 1$, then $\|T v\|_{1, p(x), w}$ is bounded.

- If $\|\nabla u\|_{p(x), w}>1$, then we have by the Proposition 2.2

$$
\|T v\|_{1, p(x), w}^{p^{-}}=\|\nabla u\|_{p(x), w}^{p-} \leq I_{p, w}(\nabla u) .
$$

By the assumption (3.3), we have

$$
a(x, \nabla u) . \nabla u \geq \alpha w(x)|\nabla u|^{p(x)} .
$$

Then

$$
\begin{aligned}
I_{p, w}(\nabla u) & =\int_{\Omega}|\nabla u|^{p(x)} w(x) d x \\
& \leq \frac{1}{\alpha} \int_{\Omega} a(x, \nabla u) . \nabla u \\
& =\frac{1}{\alpha}\langle A u, u\rangle \\
& =\frac{1}{\alpha}\langle v, T v\rangle \\
& =\frac{-t}{\alpha}\langle S o T v, T v\rangle .
\end{aligned}
$$


This implies that

$$
I_{p, w}(\nabla u) \leq \frac{t}{\alpha} \int_{\Omega} f(x, u, \nabla u) u d x .
$$

We get, by the inequalities (3.10), (3.11) and the growth condition (3.4), the estimate

$$
\|T v\|_{1, p(x), w}^{p^{-}} \leq \operatorname{const}\left(\int_{\Omega}\left|w^{\frac{1}{p(x)}} h(x) u(x)\right| d x+I_{q, w}(u)+\int_{\Omega}|\nabla u|^{q(x)-1}|u| w(x) d x\right) .
$$

Since $h \in L^{p^{\prime}(x)}(\Omega)$, then $w^{\frac{1}{p(\cdot)}} h \in L^{p^{\prime}(x)}\left(\Omega, w^{*}\right)$. By the Hölder inequality, we have

$$
\int_{\Omega}\left|w^{\frac{1}{p(x)}} h(x) u(x)\right| d x \leq 2\left\|w^{\frac{1}{p(\cdot)}} h\right\|_{p^{\prime}(x), w^{*}}\|u\|_{p(x), w}=2\|h\|_{p^{\prime}(x)}\|u\|_{p(x), w} .
$$

By the Young inequality, we have

$$
\begin{aligned}
\int_{\Omega}|\nabla u|^{q(x)-1}|u| w(x) d x & =\int_{\Omega}|\nabla u|^{q(x)-1} w^{\frac{1}{q^{\prime}(x)}} \cdot|u| w^{\frac{1}{q(x)}} d x \\
& \leq \int_{\Omega} \frac{1}{q^{\prime}(x)}|\nabla u|^{q(x)} w(x) d x+\int_{\Omega} \frac{1}{q(x)}|u|^{q(x)} w(x) d x \\
& =\frac{1}{q^{\prime-}} I_{q, w}(\nabla u)+\frac{1}{q^{-}} I_{q, w}(u) \\
& \leq \operatorname{const}\left(\|\nabla u\|_{q(x), w}^{q^{+}}+\|u\|_{q(x), w}^{q^{+}}+\|u\|_{q(x), w}^{q^{-}}\right) .
\end{aligned}
$$

We can then deduce

$$
\|T v\|_{1, p(x), w}^{p^{-}} \leq \operatorname{const}\left(\|u\|_{p(x), w}+\|u\|_{q(x), w}^{q^{+}}+\|u\|_{q(x), w}^{q^{-}}+\|\nabla u\|_{q(x), w}^{q^{+}}\right) .
$$

By the Poincaré inequality, we have

$$
\|u\|_{p(x), w} \leq C\|\nabla u\|_{p(x), w}=C\|T v\|_{1, p(x), w} .
$$

From the continuous embedding $L^{p(x)}(\Omega, w) \hookrightarrow L^{q(x)}(\Omega, w)$ and the Poincaré inequality and, we have

$$
\begin{aligned}
\|u\|_{q(x), w}^{q^{+}} & \leq C\|\nabla u\|_{q(x), w}^{q^{+}}=C\|T v\|_{1, q(x), w}^{q^{+}} \\
\|u\|_{q(x), w}^{q^{-}} & \leq C\|\nabla u\|_{q(x), w}^{q^{-}}=C\|T v\|_{1, q(x), w}^{q^{-}} \leq C\|T v\|_{1, q(x), w}^{q^{+}} \\
\|\nabla u\|_{q(x), w}^{q^{+}} & =\|T v\|_{1, q(x), w}^{q^{+}}
\end{aligned}
$$


We can then deduct the estimate

$$
\|T v\|_{1, p(x), w}^{p^{-}} \leq \operatorname{const}\left(\|T v\|_{1, p(x), w}+\|T v\|_{1, p(x), w}^{q^{+}}\right) .
$$

It follows that $\{T v \mid v \in B\}$ is bounded.

Since the operator $S$ is bounded, it is obvious from (3.9) that the set $B$ is bounded in $W^{-1, p^{\prime}(x)}\left(\Omega, w^{*}\right)$.

Consequently, there exists $R>0$ such that

$$
\|v\|_{-1, p^{\prime}(x), w^{*}}<R \text { for all } v \in B
$$

This says that

$$
v+t S o T v \neq 0 \text { for all } v \in \partial B_{R}(0) \text { and all } t \in[0,1] .
$$

From Lemma 3.4 it follows that

$$
I+S o T \in \mathcal{F}_{T}\left(\overline{B_{R}(0)}\right) \text { and } I=A o T \in \mathcal{F}_{T}\left(\overline{B_{R}(0)}\right) .
$$

Consider a homotopy $H:[0,1] \times \overline{B_{R}(0)} \rightarrow W^{-1, p^{\prime}(x)}\left(\Omega, w^{*}\right)$ given by

$$
H(t, v):=v+t S o T v \text { for }(t, v) \in[0,1] \times \overline{B_{R}(0)} .
$$

Applying the homotopy invariance and normalization property of the degree $d$ stated in Theorem 2.7, we get

$$
d\left(I+S o T, B_{R}(0), 0\right)=d\left(I, B_{R}(0), 0\right)=1,
$$

and hence there exists a point $v \in B_{R}(0)$ such that

$$
v+S o T v=0 .
$$

Finally, we conclude that $u=T v$ is a weak solution of (1.1).

\section{References}

[1] I. Aydin, "Weighted variable Sobolev spaces and capacity", Journal of function spaces and applications, vol. 2012, pp. Article ID 132690, 2012, doi: $10.1155 / 2012 / 132690$. 
[2] E. Azroul, M. Benboubker and A. Barbara, "Quasilinear elliptic problems with nonstandard growth", Electronic journal of differential equations, vol. 2011, no. 62, pp. 1-16, 2011. [On line]. Available: https://bitly/2BewUWI

[3] J. Berkovits, On the degree theory for nonlinear mappings of monotone type, vol. 58. Helsinki: Suomalainen Tiedeakatemia, 1986.

[4] J. Berkovits, "Extension of the Leray-Schauder degree for abstract Hammerstein type mappings", Journal of differential equations, vol. 234, no. 1, pp. 289-310, Mar. 2007, doi: 10.1016/j.jde.2006.11.012

[5] J. Berkovits, and V. Mustonen, "On topological degree for mappings of monotone type", Nonlinear analysis, vol. 10, no. 12, pp. 1373-1383, Dec. 1986, doi: 10.1016/0362-546X(86)90108-2

[6] J. Berkovits and V. Mustonen, Nonlinear mappings of monotone type I. Classification and degree theory, Preprint no 2/88, Mathematics, University of Oulu, 1988.

[7] M. Boureanu, A. Matei and M. Sofonea, "Nonlinear problems with p(.)growth conditions and applications to antiplane contact models", Advanced nonlinear studies, vol. 14, no. 2, pp. 295-313, May 2014, doi: 10.1515/ans-2014-0203.

[8] L. Brouwer, "Uber abbildung von mannigfaltigkeiten", Mathematische annalen, vol. 71, no. 1, pp. 97-115, Mar. 1912, doi: 10.1007/BF01456931.

[9] L. Diening, P. Harjulehto, P. Hästö, and M. Ruzicka, Lebesgue and Sobolev spaces with variable exponents, vol. 2017. Berlin: Springer, 2011, doi: 10.1007/978-3-642-18363-8.

[10]X. Fan and Q. Zhang, "Existence for p(x)-Laplacien Dirichlet problem", Non linear analysis, vol. 52, no. 8, pp. 1843-1852, Mar. 2003, doi: 10.1016/S0362-546X(02)00150-5.

[11]X. Fan, D. Zhao, "On the spaces $\operatorname{Lp}(\mathrm{x})(\Omega)$ and Wm,p $(\mathrm{x})(\Omega)$ ”, Journal of mathematical analysis and applications, vol. 263, no. 2, pp. 424-446, Nov. 2001, doi: 10.1006/jmaa.2000.7617.

[12] 0. Kováčik and J. Rákosník, "On spaces Lp(x) and Wk,p(x)”, Czechoslovak mathematical journal, vol. 41, no. 4. pp. 592-618, 1991 [On line]. Available: http://bit.ly/2VQzJqg

[13]B. Lahmi, E. Azroul and K. El Haiti, "Nonlinear degenerated elliptic problems with dual data and nonstandard growth", Mathematical reports, vol. 20, no. 1, pp. 81-91, 2018. [On line]. Available: http://bit.ly/31u0pi8

[14]J. Leray and J. Schauder, "Topologie et équations fonctionnelles", Annales scientifiques de lÉcole normale supérieure. 3e série, vol. 51, pp. 45-78, 1934, doi: 10.24033 /asens.836

[15] K. Rajagopal and M. Ružička, "Mathematical modeling of electrorheological materials", Continuum mechanics and thermodynamics, vol. 13, no. 1, pp. 59-78, Jan. 2001, doi: 10.1007/s001610100034. 
[16] M. Ružička, Electrorheological fluids: modeling and mathematical theory, vol. 1748. Berlin: Springer, 2000, doi: 10.1007/BFB0104029.

[17] [S. Samko, "Density of $\mathrm{C}_{0}{ }^{\infty} 0\left(\mathrm{R}^{\mathrm{n}}\right)$ in the generalized Sobolev spaces $\mathrm{W}^{\mathrm{m}, \mathrm{p}(\mathrm{x})}\left(\mathrm{R}^{\mathrm{n}}\right)^{\prime}$, Doklady akademii nauk, vol. 369 , no, pp. 451-454, 1999. [On line]. Available: http://bit.ly/32oJHSj

[18] M. Sanchon, J. Urbano, "Entropy solutions for the $\mathrm{p}(\mathrm{x})$-Laplace equation", Transactions of the american mathematical society, vol. 361, no. 12, pp. 6387-6405, 2009, doi: 10.1090/S0002-9947-0904399-2.

[19] C. Unal and I. Aydin, "Weighted variable exponent Sobolev spaces with zero boundary values and capacity estimates", Sigma journal of engineering and natural sciences, vol. 36, no. 2, pp. 373-388, 2018. [On line]. Available: http://bit.ly/2VTUHVA

[20] C. Unal and I. Aydin, "Compact embedding on a subspace of weighted variable exponent Sobolev spaces", Advances in operator theory, vol. 4, no. 2, pp. 388-405, 2019, doi: 10.15352/A0T.18031335

[21] E. Zeidler, Nonlinear monotone operators, vol. 2-B. New York, NY: Springer, 1990, doi: 10.1007/978-1-4612-0981-2.

[22] V. Zhikov, "Averaging of functionals of the calculus of variations and elasticity theory", Mathematics of the USSR-Izvestiya, vol. 29, no. 1, pp. 33-66, 1987, doi: 10.1070/IM1987v029n01ABEH000958 